\title{
Coulometrischer Sensor für die Niederdruckaufkohlung
}

\author{
J. Zosel ${ }^{1)}$, V. Vashook ${ }^{2}$, H.-J. Berg ${ }^{3)}$, W. Gräfen ${ }^{3)}$, U. Guth ${ }^{1,2)}$
}

1) Kurt-Schwabe-Institut für Mess- und Sensortechnik e.V. Meinsberg, Kurt-Schwabe-Straße 4, 04720 Ziegra-Knobelsdorf

2) Technische Universität Dresden, Fachrichtung Chemie und Lebensmittelchemie, 01062 Dresden

3) Hanomag Härtol Gommern Lohnhärterei GmbH, Magdeburger Straße 21, 39245 Gommern

\section{Zusammenfassung}

Für die zeitnahe Regelung von Anlagen zur Niederdruckaufkohlung mit Ethin werden robuste Sensoren und Messsysteme benötigt, die ein Signal zu dem momentan in den Werkstoff übergehenden Kohlenstoff liefern. Für diese Applikation wurde ein coulometrischer Festelektrolytsensor auf der Basis von stabilisiertem Zirconiumdioxid eingesetzt, der die kontinuierliche Umsetzung eines Teilstroms des Ofenabgases mit Sauerstoff ermöglicht. Untersuchungen im Labor und an einer Anlage zeigen, dass der Titrationsstrom dann als direktes Maß für den Kohlenstoffübergang herangezogen werden kann.

\section{Einleitung und Zielstellung}

Die schnelle Regelung von Anlagen zur Niederdruckaufkohlung [1] gewinnt zunehmend an Bedeutung. Dazu werden robuste Sensoren und Messsysteme benötigt, die in Anlagen zur Niederdruckaufkohlung (NDA) eingesetzt werden können, in denen metallische Werkstücke bei Drücken zwischen 2 . 10 mbar und Temperaturen zwischen $950 \ldots 1100{ }^{\circ} \mathrm{C}$ in einer Ethin-haltigen Atmosphäre gehärtet werden. Insbesondere wird ein Signal benötigt, mit dem sich der momentan in den Werkstoff übergehende Kohlenstoff direkt beurteilen lässt. Eine wesentliche Besonderheit des Prozesses besteht in der pulsartigen Einführung des Ethins, wobei die Pulsdauer $2 \ldots 8$ min und die Pulspausen $5 \ldots 60$ min betragen können. In den Pulspausen wird das Restgas abgesaugt, während der durch Zerfall während des Ethinpulses an der Werkstoffoberfläche abgelagerte Kohlenstoff in die Tiefe diffundiert [2]. Um die Kohlenstoffdosierung geeignet beurteilen und regeln zu können, muss demnach während eines Ethinpulses der Kohlenstoffübergang mit Ansprechzeiten $t_{90}<10 \mathrm{~s}$ gemessen werden. In der Vergangenheit wurden bereits vereinzelt Wasserstoffsensoren auf der Basis der Wärmeleitfähigkeit für diese Applikation eingesetzt [3]. Diese können nicht die bei der Niederdruckaufkohlung entstehenden Zwischenprodukte erkennen, liefern nur indirekte Informationen und sind deshalb prinzipbedingt fehlerbehaftet. Außerdem weisen sie bislang noch zu hohe Ansprechzeiten für diesen Einsatzzweck auf. Ausgehend vom Stand der Technik bestand das Ziel dieser Arbeit in der Entwicklung, Herstellung und Erprobung eines Messsystems, mit dem der Kohlenstoffübergang direkt mit kurzer Ansprechzeit am Prozess gemessen werden kann.

\section{Sensorprinzip}

Ein Grundprinzip coulometrischer Festelektrolytsensoren beruht, wie in Bild 1 gezeigt, auf der Messung des Stoffumsatzes an einer Elektrolyse-Elektrode in Kombination mit der quasi-simultanen potentiometrischen Bestimmung des Partialdrucks von Sauerstoff, der als Ion durch den Festelektrolyt transportiert wird. Stabilisiertes Zirconiumdioxid (YSZ) als Sauerstoffionenleiter und das katalytisch hochaktive Platin als Elektrodenmaterial ermöglichen neben der Sauerstoffmessung auch die Erfassung oxidierbarer Gase in sauerstofffreien Gemischen, wie sie bei der Niederdruckaufkohlung vorliegen. Das Ofenabgas, bestehend aus einem Gemisch aus Ethin, Wasserstoff und einer Reihe von Pyrolyseprodukten [4] der Summenformel $\mathrm{C}_{\mathrm{x}} \mathrm{H}_{\mathrm{y}}$, wird gemäß folgender Gleichung (1) in einem coulometrischen Sensor mit Sauerstoffionen umgesetzt. 
Die Koeffizienten $\mathrm{k}, \mathrm{m}$ und $\mathrm{n}$ hängen dabei vor allem von dem bereits auf der Werkstückoberfläche vorhandenen Kohlenstoff und den katalytischen Eigenschaften der Werkstïckoberfläche ab.

Das Ofenabgas wird durch den Sensor mit einer Vakuumpumpe gesaugt, wobei der Volumenstrom durch eine Kapillare mit $\mathrm{d}=0,1 \mathrm{~mm}$ und $\mathrm{l}=70 \mathrm{~mm}$ begrenzt wird.

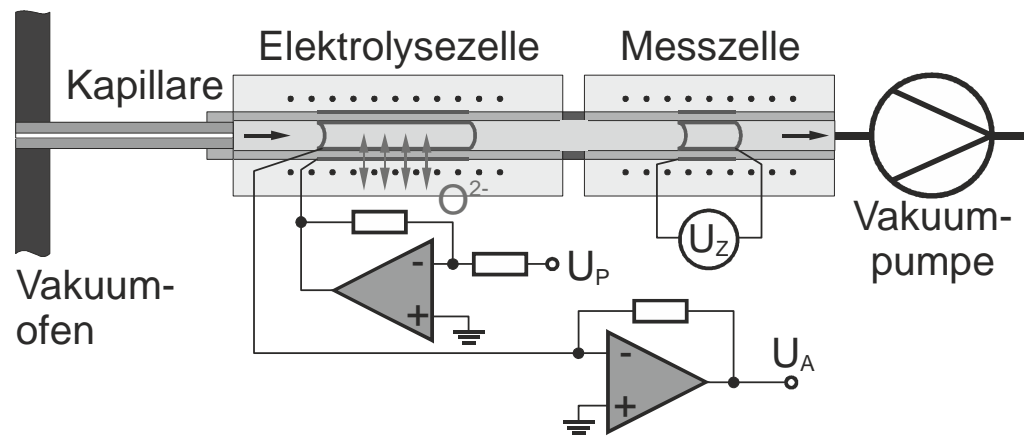

Bild 1 Schematische Darstellung des coulometrischen Festelektrolytsensors für die Regelung der Niederdruckaufkohlung mit Ethin

\section{Ergebnisse}

\subsection{Sensorcharakterisierung im Labor}

Zur Untersuchung der Eignung des neuentwickelten Messsystems für die geplante Applikation wurden dessen Parameter im Labor unter Einsatz von Ethin-Wasserstoff-Gemischen durchgeführt, die aus einem Vakuum-Reaktor mit V = $16 \mathrm{ml}$ abgezogen wurden. Die in Bild 2 aufgeführten Kurven für den Elektrolysestrom belegen, dass dieser beim Übergang von $\mathrm{H}_{2} \mathrm{zu} \mathrm{C}_{2} \mathrm{H}_{2}$ im Reaktor nicht wie gemäß Gleichung $1 \mathrm{zu}$ erwarten ist, um den Faktor fünf ansteigt. Die gemessenen Faktoren sind vom Druck abhängig und weisen im untersuchten Druckbereich Werte zwischen 1,8 .. 2,1 auf. Dieses Resultat wird vor allem durch die unterschiedlichen Diffusionskoeffizienten und Viskositäten der beiden Gase verursacht, die den Durchgang durch die Kapillare beeinflussen. Die in Bild 2 gezeigten Kalibrierkurven ließen sich nach mehr als zehn der im folgenden Abschnitt beschriebenen Zyklen in einer Einkammeranlage reproduzieren.

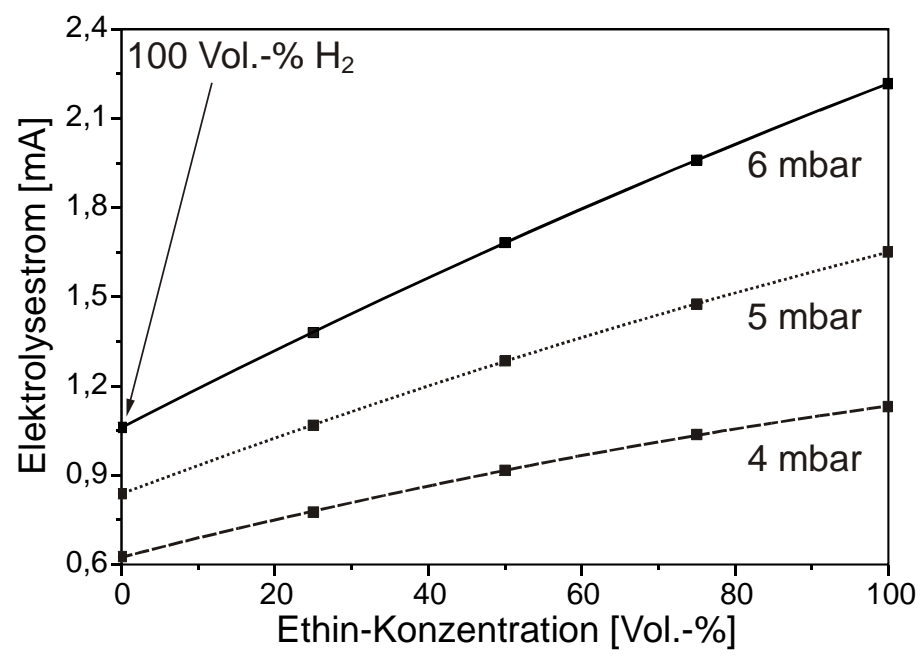

Bild 2 Elektrolysestrom des coulometrischen Sensors in Abhängigkeit von Reaktordruck und Ethinkonzentration bei Einleitung von Ethin/Wasserstoff-Gemischen in den Reaktor, Sensortemperatur $750{ }^{\circ} \mathrm{C}, \mathrm{U}_{\mathrm{P}}=-400 \mathrm{mV}$

\subsection{Erprobung an einer Anlage zur Niederdruckaufkohlung}

Die im Labor durchgeführte Kalibrierung des Sensors in Ethin/Wasserstoff-Gemischen wurde bei verschiedenen Drücken unter Einleitung von reinem $\mathrm{H}_{2}$ bzw. reinem $\mathrm{C}_{2} \mathrm{H}_{2}$ in den in Bild 3 gezeigten Ofen wiederholt. Wie in Bild 4 dargestellt, führten diese Messungen zu niedrigeren Elektrolyseströmen als die in 
Abschnitt 3.1 aufgeführten Untersuchungen im Labor. Diese Verringerung wird vor allem durch einen Anteil von Stickstoff in der Ofenatmosphäre verursacht, der auf den durch längere Evakuierung erreichbaren Enddruck zurückzuführen ist. Außerdem ist an den Großanlagen mit einer geringfügigen Eindiffusion von Sauerstoff zu rechnen, die im Labor nicht auftritt. Der Vergleich der Resultate für Ethin im heißen bzw. kalten Vakuumofen zeigt, dass Ethin bereits im leeren Ofen bei Drücken oberhalb von 4 mbar beginnt, anteilig zu zerfallen. Der dadurch freigesetzte Kohlenstoff verbleibt im Ofen und belegt die zur Verfügung stehenden Oberflächen der Heizung und der weiteren Einbauten. Mit dem neuentwickelten Sensor lässt sich dieser unerwünschte Zerfall erstmals präzise quantifizieren.

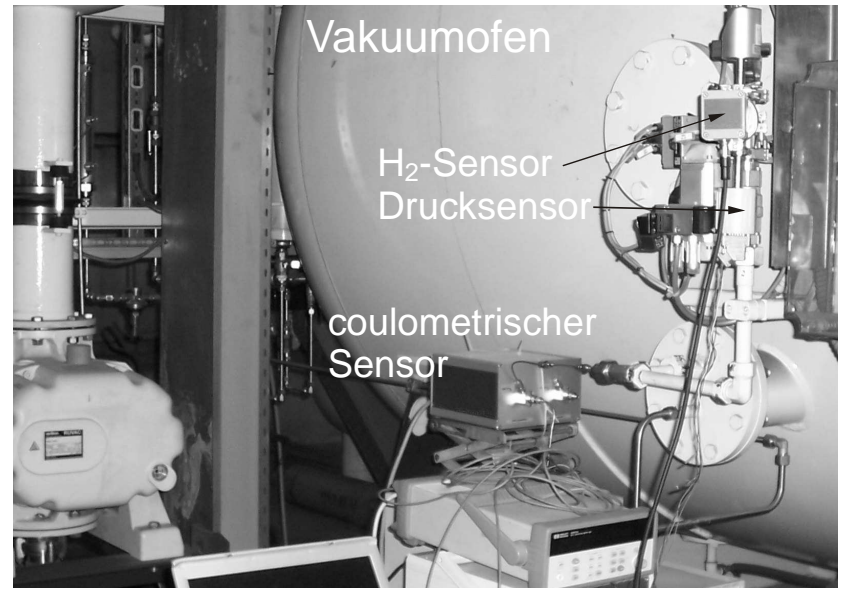

Bild 3 Anordnung des Messsystems an einer Ein-kammeranlage zur Niederdruckaufkohlung

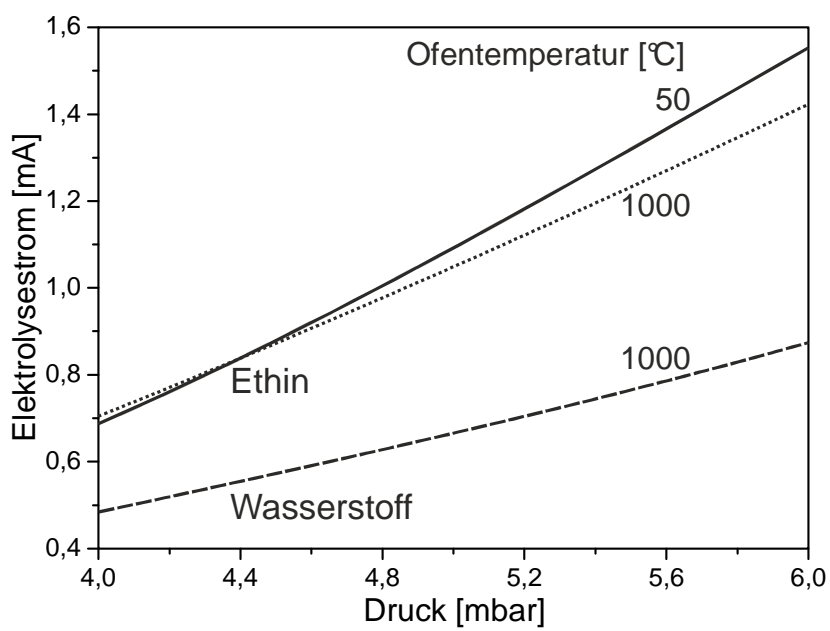

Bild 4 Elektrolysestrom in Abhängigkeit von Druck, Gasfüllung und Ofentemperatur an einer Einkammeranlage zur Niederdruckaufkohlung gemäß Bild 3

Nach der Charakterisierung des Sensors an der leeren Anlage erfolgte die Erprobung im Prozess. Dazu wurden der Ofen mit Chargiergut befüllt und reale Zyklen zur Aufkohlung der Werkstücke durchgeführt. Zusätzlich zu dem neuentwickelten coulometrischen Sensor wurde ein Wärmeleitfähigkeitssensor (WLD) an die Anlage angebaut und damit die Wasserstoffkonzentration in der Ofenatmosphäre bestimmt. Der in Bild 5 gezeigte Ausschnitt aus einem solchen Zyklus betrifft drei Ethinpulse mit je 5 min Dauer, eine längere Pulspause von 10 min sowie drei Pulse mit je 3 min Pulszeit und enthält die Signalverläufe des Druck- bzw. des coulometrischen Sensors. Der Elektrolysestrom zeigt qualitativ, wie der Kohlenstoffübergang sowohl während eines einzelnen Ethinpulses als auch von Puls zu Puls abnimmt.

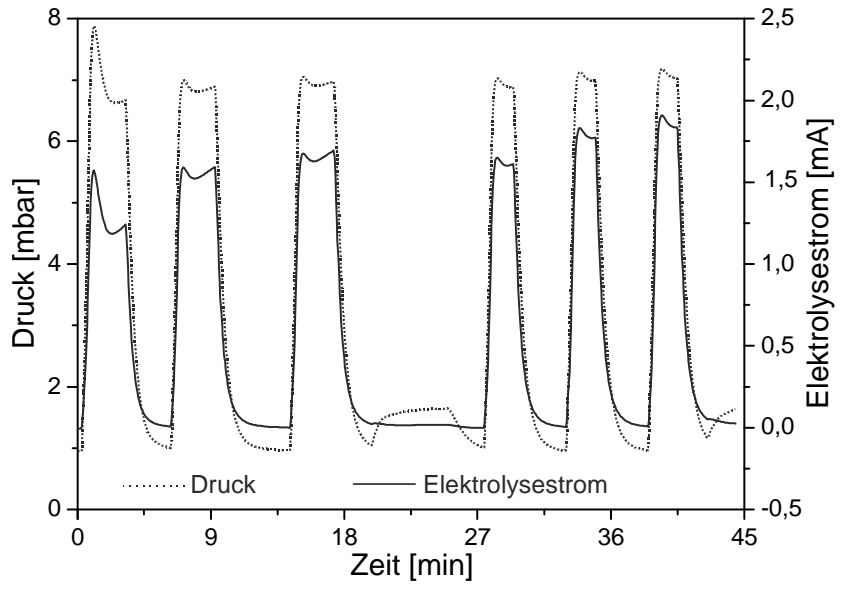

Bild 5 Verlauf von Druck und Elektrolysestrom während eines Aufkohlungszyklus mit 6 Ethinpulsen

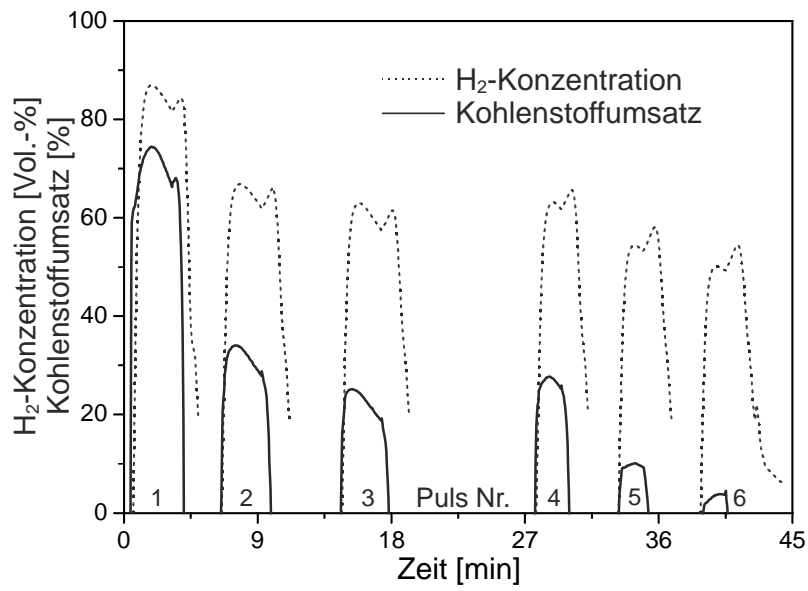

Bild $6 \mathrm{H}_{2}$-Konzentration und Kohlenstoffübergang, berechnet aus den Signalen des WLD bzw. der Elektrolysezelle gemäß Bild 5 
Die Signale des WLD und des coulometrischen Sensors wurden mit dem Signal des Drucksensors und den Daten gemäß Bild 4 in die entsprechende Wasserstoffkonzentration bzw. den prozentualen Kohlenstoffübergang umgerechnet. Die in Bild 6 dargestellten Ergebnisse belegen, dass der WLD deutlich langsamer anspricht als der coulometrische Sensor, wie beispielsweise an den Zeitpunkten der Maxima des Kohlenstoffübergangs im ersten Drittel der Pulse eins bis drei ablesbar ist. Das Signal des WLD zeigt in jedem Puls einen signifikant höheren Kohlenstoffeintrag in das Material an, als der Elektrolysestrom. Diese Diskrepanz ist neben dem spontanen Ethinzerfall an den heißen Oberflächen im Ofen außerhalb der Werkstücke (siehe auch Bild 4) vor allem auf die Kalibrierung des WLD zurückzuführen, die in $\mathrm{H}_{2} / \mathrm{N}_{2^{-}}$ Gemischen erfolgt. In der Ofenatmosphäre ist jedoch kaum $\mathrm{N}_{2}$ vorhanden. Neben $\mathrm{H}_{2}$ treten vor allem $\mathrm{C}_{2} \mathrm{H}_{2}$ und dessen Pyrolyseprodukte auf, die andere Wärmeleitfähigkeiten als $\mathrm{N}_{2}$ aufweisen. Der erneute Anstieg der $\mathrm{H}_{2}$-Konzentration im zweiten Drittel des Pulses ist auf den hier vorliegenden hohen zeitlichen Druckgradienten zurückzuführen. Dies belegt, dass die relativ hohe Differenz zwischen den Ansprechzeiten des WLD und des Drucksensors bei hohen Druckgradienten zu großen Fehlern bei der Berechnung der Wasserstoffkonzentration führt. Da zwischen den Pulsen der Druck Werte außerhalb des kalibrierten Bereiches des WLD aufweist, wurden die hier berechneten Ergebnisse in Bild 6 ausgeblendet. Aus dem Zeitintegral des momentanen Kohlenstoffübergangs und der zugeführten Ethinmenge kann der gesamte im Ofen verbliebene Kohlenstoff berechnet werden. Durch Vergleiche mit Offline-Analysen des im Werkstoff nach der Behandlung vorhandenen Kohlenstoffs bei einer Vielzahl verschiedener Chargen und Werkstoffe soll zukünftig die Reproduzierbarkeit der Messmethode charakterisiert werden.

\section{Zusammenfassung}

Ein coulometrischer Sensor auf der Basis von stabilisiertem Zirconiumdioxid wurde erstmals erfolgreich für die Online-Messung des Kohlenstoffübergangs beim Prozess der Niederdruckaufkohlung metallischer Werkstücke mit Ethin eingesetzt. Messungen über mehrere Wochen beeinträchtigten weder die Elektrodenaktivität noch die fluidischen Eigenschaften der Kapillare. Untersuchungen an einer Einkammeranlage belegen, dass der neuentwickelte Sensor eine kürzere Ansprechzeit als die bislang für die Regelung solcher Prozesse vereinzelt eingesetzten Wärmeleitfähigkeitsdetektoren (WLD) aufweist. Da diese vor allem auf den in der Ofenatmosphäre enthaltenen Wasserstoff ansprechen, der Elektrolysestrom des coulometrischen Sensors im Gegensatz dazu ein Maß für den im Abgas enthaltenen Kohlenstoff liefert, lässt sich der Kohlenstoffübergang mit der neuen Methode direkt messen, wobei wesentliche Fehlerquellen der WLD vermeidbar sind. Nach dem Funktionsnachweis an einer Praxisanlage sind Felduntersuchungen zur Reproduzierbarkeit und Übertragbarkeit auf andere Anlagen vorgesehen.

\section{Literatur}

[1] W. Gräfen, B. Edenhofer: New developments in thermo-chemical diffusion processes, Surface \& Coatings Technology 200 (2005) 1830-1836.

[2] W. Gräfen: Untersuchungen zum Kohlenstoffübergang bei der Niederdruckaufkohlung, Aachen: Shaker Verlag 2002.

[3] H. Altena, F. Schrank, S. Heineck: Prozessüberwachung und Regelung von Niederdruck-Aufkohlungsprozessen, HTM 61 (2006) 4, 195-205.

[4] P. Jacquet, G. Bernard, M. Lambertin: A new sensor to follow low pressure carburizing. In S. J. Midea, G. D. Pfaffmann, Proc. of the $19^{\text {th }}$ Conference of Heat Treating, ASM International (2000) 187-189.

\section{Danksagung}

Teile der vorgestellten Arbeit wurden mit Mitteln des Bundesministeriums für Wirtschaft und Technologie unter dem Förderkennzeichen EP100441 gefördert. Die Autoren danken für die Förderung. 